\title{
Classification of lossless first-order optical systems and the linear canonical transformation
}

\author{
Martin J. Bastiaans \\ Faculteit Elektrotechniek, Technische Universiteit Eindhoven, Postbus 513, 5600 MB Eindhoven, Netherlands
}

Tatiana Alieva

Facultad de Ciencias Físicas, Universidad Complutense de Madrid, Ciudad Universitaria s/n, Madrid 28040, Spain

Received July 18, 2006; revised October 27, 2006; accepted November 10, 2006; posted November 13, 2006 (Doc. ID 73122); published March 14, 2007

\begin{abstract}
Based on the eigenvalues of the ray transformation matrix, a classification of ABCD systems is proposed and some nuclei (i.e., elementary members) in each class are described. In the one-dimensional case, possible nuclei are the magnifier, the lens, and the fractional Fourier transformer. In the two-dimensional case we have-in addition to the obvious concatenations of one-dimensional nuclei-the four combinations of a magnifier or a lens with a rotator or a shearing operator, where the rotator and the shearer are obviously inherently twodimensional. Any ABCD system belongs to one of the classes described in this paper and is similar (in the sense of matrix similarity of the ray transformation matrices) to the corresponding nucleus. Knowledge of a nucleus may be helpful in finding eigenfunctions of the corresponding class of first-order optical systems: one only has to find eigenfunctions of the nucleus and to determine how these functions propagate through a firstorder optical system. (C) 2007 Optical Society of America
\end{abstract}

OCIS codes: $070.2580,070.4690,080.2730,120.4820$.

\section{INTRODUCTION}

Many papers have been published about lossless firstorder optical systems, ${ }^{1-6}$ also known as ABCD systems, and the linear canonical integral transformation ${ }^{7-9}$ associated to these systems. There seems, however, to be no complete overview of the different classes that can be distinguished for such systems, except for a paper by Pei and Ding, ${ }^{10}$ in which the one-dimensional case was treated. In this paper we will consider the two-dimensional case, i.e., lossless first-order optical systems with two transverse coordinates, $x$ and $y$, combined into a two-dimensional column vector $\mathbf{r}=(x, y)^{t}$, and a $4 \times 4$ ray transformation matrix $\mathbf{T}$, also known as the ABCD matrix, through which such a system can be described. We recall that the ray transformation matrix $\mathbf{T}$ is real and symplectic.

Our classification will be based on the distribution of the eigenvalues of the ray transformation matrix, and on whether or not this matrix is diagonalizable. Our method will lead to three classes for the one-dimensional case and four additional classes for the two-dimensional case. For each class we will determine a basic system (a nucleus) that satisfies the properties of a lossless first-order optical system (realness and symplecticity) and that is described by the lowest possible number of parameters. For the onedimensional case we will thus find such well-known systems as the magnifier (or the hyperbolic expander; see, for example, Ref. 6, p. 183, Example: Hyperbolic expanders), the thin lens (or a section of free space), and the optical fractional Fourier transformer. ${ }^{11-16}$ The two-dimensional case leads to some additional nuclei: combinations of an isotropic magnifier (hyperbolic expander) or an isotropic lens (section of free space) with a rotator or a shearer.
Once the nucleus is known, the ray transformation matrix of a system that belongs to a certain class is similar to the ray transformation matrix of the corresponding nucleus. So, we can write $\mathbf{T}=\mathbf{M} \mathbf{T}_{n} \mathbf{M}^{-1}$, where $\mathbf{T}_{n}$ is the ray transformation matrix of the nucleus; moreover, $\mathbf{M}$ is also a proper ray transformation matrix, associated to a lossless first-order optical system. We remark that this similarity relation resembles the one that would arise if we try to bring the matrix $\mathbf{T}$ into Jordan form, ${ }^{17}$ but the problem with the Jordan form is that it is not guaranteed that the properties of realness and symplecticity are preserved. A proof for the fact that $\mathbf{M}$ can be chosen to be a proper ray transformation matrix can be given for each separate class that will be considered; see, for instance, Ref. 18 for the class of unimodular eigenvalues. A general proof can be found in Ref. 19; see, in particular, Theorem 41 in Ref. 19, which deals with the real symplectic Jordan form.

From the similarity relation $\mathbf{T}=\mathbf{M} \mathbf{T}_{n} \mathbf{M}^{-1}$, we easily conclude that if $\Phi(\mathbf{r})$ is an eigenfunction of the nucleus, then the function $\Phi^{\prime}(\mathbf{r})$, say, that arises when we let $\Phi(\mathbf{r})$ propagate through the system with ray transformation matrix $\mathbf{M}, \Phi(\mathbf{r}) \rightarrow \Phi^{\prime}(\mathbf{r})$, is an eigenfunction of the cascade $\mathbf{T}=\mathbf{M} \mathbf{T}_{n} \mathbf{M}^{-1}$. This may be immediately clear from the following: when the function $\Phi^{\prime}(\mathbf{r})$ is chosen as the input function of the cascade $\mathbf{M} \mathbf{T}_{n} \mathbf{M}^{-1}$, the inverse $\mathbf{M}$ system will convert it into $\Phi(\mathbf{r})$, which then acts as an eigenfunction of the nucleus $\mathbf{T}_{n}$, after which the forward $\mathbf{M}$ system transforms $\Phi(\mathbf{r})$ back into the function $\Phi^{\prime}(\mathbf{r})$ with which we started. But for this to work, the ray transformation matrix $\mathbf{M}$ should be real and symplectic, which is why we cannot simply use the Jordan form, but often need to find 
a proper nucleus instead. Knowledge of the nucleus $\mathbf{T}_{n}$ may then be helpful in finding eigenfunctions of the corresponding class of first-order optical systems, decomposable as $\mathbf{M} \mathbf{T}_{n} \mathbf{M}^{-1}$.

The paper is organized as follows. We first recall the basic description of a lossless first-order optical system by means of its real and symplectic ray transformation matrix $\mathbf{T}$ in Section 2. In Section 3 we then consider the different possible distributions of the eigenvalues of the real symplectic matrix $\mathbf{T}$, in connection with whether or not $\mathbf{T}$ can be diagonalized. The main work is done in Sections 4 and 5, where for each class of eigenvalue distributions the corresponding nucleus will be formulated. We will present the three one-dimensional classes in Section 4 and the four additional two-dimensional classes in Section 5. In Section 6 we will show how, from the knowledge of the different nuclei, eigenfunctions for the different classes can be found.

\section{FIRST-ORDER OPTICAL SYSTEMS}

Any lossless first-order optical system can be described by its ray transformation matrix, ${ }^{1,5}$ which relates the position $\mathbf{r}_{i}$ and direction (or, maybe better, spatial frequency, which is proportional to the transversal part of the ray vector; see the remark at the end of this section) $\mathbf{p}_{i}$ of an incoming ray to the position $\mathbf{r}_{o}$ and direction $\mathbf{p}_{o}$ of the outgoing ray:

$$
\left[\begin{array}{l}
\mathbf{r}_{o} \\
\mathbf{p}_{o}
\end{array}\right]=\mathbf{T}\left[\begin{array}{l}
\mathbf{r}_{i} \\
\mathbf{p}_{i}
\end{array}\right]=\left[\begin{array}{ll}
\mathbf{A} & \mathbf{B} \\
\mathbf{C} & \mathbf{D}
\end{array}\right]\left[\begin{array}{l}
\mathbf{r}_{i} \\
\mathbf{p}_{i}
\end{array}\right]
$$

The vectors $\mathbf{r}$ and $\mathbf{p}$ are in general $D$-dimensional column vectors, the ray transformation matrix $\mathbf{T}$ is $2 D \times 2 D$, and all block matrices are $D \times D$; in this paper we will restrict ourselves to the cases $D=1$ and $D=2$. The ray transformation matrix of such a system is real and symplectic. Symplecticity can be expressed elegantly in the form

$$
\left[\begin{array}{ll}
\mathbf{A} & \mathbf{B} \\
\mathbf{C} & \mathbf{D}
\end{array}\right]^{-1}=\left[\begin{array}{cc}
\mathbf{D}^{t} & -\mathbf{B}^{t} \\
-\mathbf{C}^{t} & \mathbf{A}^{t}
\end{array}\right] \text { or } \quad \mathbf{T}^{-1}=\mathbf{J} \mathbf{T}^{t} \mathbf{J}
$$

with

$$
\mathbf{J}=i\left[\begin{array}{cc}
\mathbf{0} & -\mathbf{I} \\
\mathbf{I} & \mathbf{0}
\end{array}\right], \quad \mathbf{J}=\mathbf{J}^{-1}=\mathbf{J}^{\dagger}=-\mathbf{J}^{t},
$$

where $\mathbf{I}$ is the identity matrix and $\mathbf{0}$ the null matrix; as usual, the superscript $t$ denotes transposition, and the superscript $\dagger$ is used to denote the combined action of transposition and complex conjugation. As some well-known one-dimensional examples we mention the matrices

$$
\mathbf{T}_{\mathcal{L}}(f)=\left[\begin{array}{cc}
1 & 0 \\
-1 / \lambda_{0} f & 1
\end{array}\right], \quad \mathbf{T}_{\mathcal{S}}(z)=\left[\begin{array}{cc}
1 & \lambda_{0} z \\
0 & 1
\end{array}\right]
$$

(see Ref. 5, Appendixes B.3 and B.4), corresponding to a lens $\mathcal{L}(f)$ with focal length $f$ and to a section of free space $\mathcal{S}(z)$ with distance $z$, acting on light with wavelength $\lambda_{0}$; and, in particular, the matrix ${ }^{13}$

$$
\mathbf{T}_{\mathcal{F}}(\theta ; w)=\left[\begin{array}{cc}
\cos \theta & w^{2} \sin \theta \\
-w^{-2} \sin \theta & \cos \theta
\end{array}\right],
$$

corresponding to a fractional Fourier transformer ${ }^{13-16}$ $\mathcal{F}(\theta ; w)$ with fractional angle $\theta$ and scaling $w$.

We recall that in the one-dimensional case, with transversal coordinate $x$ and longitudinal coordinate $z$, the spatial frequency $p_{x}$ is equal to $\sin \varphi_{x} / \lambda_{0}$, where $\varphi_{x}$ is the angle (in the $x z$ plane) between the ray and the $z$ axis. The extension to two dimensions, with $\mathbf{r}=(x, y)^{t}$ being the transversal coordinates, is straightforward; we now have two angles, $\varphi_{x}$ and $\varphi_{y}$, and the spatial frequency takes the form $\mathbf{p}=\left(p_{x}, p_{y}\right)^{t}=\left(\sin \varphi_{x} / \lambda_{o}, \sin \varphi_{y} / \lambda_{0}\right)^{t}$. We finally note that, unlike common practice (see, for example, Ref. 5, Appendixes B.3 and B.4), we do not use reduced quantities, i.e., we do not divide by the index of refraction.

\section{EIGENVALUES OF REAL SYMPLECTIC MATRICES}

In this paper we propose a classification of first-order optical systems, based on the eigenvalues of the ray transformation matrix. We recall ${ }^{18}$ that if $\lambda$ is an eigenvalue of a real symplectic matrix $\mathbf{T}$, then $\lambda^{*}, 1 / \lambda$, and $1 / \lambda^{*}$ are eigenvalues, too; as usual, complex conjugation is denoted by the superscript $*$. Indeed, from the realness of $\mathbf{T}$, we conclude that the characteristic equation $\operatorname{det}(\mathbf{T}-\lambda \mathbf{I})=0$ has real coefficients and that the eigenvalues are thus real or come in complex conjugated pairs: if $\lambda$ is an eigenvalue, then $\lambda^{*}$ is an eigenvalue, too. Moreover, from the symplecticity condition [Eq. (2)] we get

$$
\begin{aligned}
\operatorname{det}\left(\mathbf{T}^{-1}-\lambda \mathbf{I}\right) & =\operatorname{det}\left(\mathbf{J T}^{t} \mathbf{J}-\lambda \mathbf{I}\right)=\operatorname{det}\left[\mathbf{J}\left(\mathbf{T}^{t}-\lambda \mathbf{I}\right) \mathbf{J}\right] \\
& =\operatorname{det}\left(\mathbf{T}^{t}-\lambda \mathbf{I}\right)=\operatorname{det}(\mathbf{T}-\lambda \mathbf{I})
\end{aligned}
$$

and we conclude that if $\lambda$ is an eigenvalue, then $1 / \lambda$ is an eigenvalue, too. So, for real symplectic matrices and $D$ $\geq 2$, the eigenvalues come in complex quartets (if they are not unimodular and not real), or in complex conjugated pairs (if they are unimodular, but not real), or in real pairs (in particular, double if equal to +1 or -1 ). For $D$ $=1$, the two eigenvalues can, of course, only come as a single pair, either unimodular or real.

Each matrix is similar to a Jordan matrix $\Delta$ (Ref. 17): $\mathbf{T}=\mathbf{Q} \boldsymbol{\Delta} \mathbf{Q}^{-1}$. The Jordan matrix $\boldsymbol{\Delta}$ has the eigenvalues of $\mathbf{T}$ on its main diagonal, and may or may not have additional off-diagonal entries. In the case that $\mathbf{T}$ can be diagonalized, its Jordan matrix $\Delta$ does not contain any additional off-diagonal entries, and the columns of $\mathbf{Q}$ constitute a set of $2 D$ linearly independent eigenvectors of $\mathbf{T}$. In the case that $\mathbf{T}$ cannot be diagonalized (which is the same as saying that $\mathbf{T}$ does not have a set of $2 D$ linearly independent eigenvectors), the Jordan matrix will contain off-diagonal entries, and not all columns of $\mathbf{Q}$ are eigenvectors of $\mathbf{T}$. Let us consider the possibilities for $D=1$ and $D=2$. For $D=1$ we have the following three cases.

1. A pair of real eigenvalues, $s$ and $s^{-1}(s \neq \pm 1)$, with two linearly independent eigenvectors; the Jordan matrix reads 


$$
\Lambda_{\mathcal{M}}(s)=\left[\begin{array}{cc}
s & 0 \\
0 & s^{-1}
\end{array}\right] \text {. }
$$

This case will be treated in Subsection 4.A. We will consider the special unimodular case $s=\exp (i k \pi)$ as belonging to case 3.

2 . Two real eigenvalues, $\lambda=1$ or $\lambda=-1$, with only one eigenvector; the Jordan matrix reads

$$
\mathbf{J}_{+}(\lambda)=\left[\begin{array}{ll}
\lambda & 1 \\
0 & \lambda
\end{array}\right] \quad \text { or } \quad \mathbf{J}_{-}(\lambda)=\left[\begin{array}{cc}
\lambda & 0 \\
-1 & \lambda
\end{array}\right] .
$$

This case will be treated in Subsection 4.B. For convenience, when $\lambda=1$ we will simply write the shorthand forms $\mathbf{J}_{+}=\mathbf{J}_{+}(1)$ and $\mathbf{J}_{-}=\mathbf{J}_{-}(1)$.

3. A pair of two unimodular, complex conjugated eigenvalues $\exp (i \theta)$ and $\exp (-i \theta)$, with two linearly independent eigenvectors; the Jordan matrix reads

$$
\Lambda_{\mathcal{F}}(\theta)=\left[\begin{array}{cc}
\exp (i \theta) & 0 \\
0 & \exp (-i \theta)
\end{array}\right] .
$$

This case will be treated in Subsection 4.C. Although the matrix in Eq. (8) resembles the one in Eq. (6), we will treat the two cases separately. For $D=2$ we have, of course, concatenations of the one-dimensional Jordan matrices, where the concatenation operator $\oplus$ is defined as

$$
\left[\begin{array}{ll}
a_{1} & b_{1} \\
c_{1} & d_{1}
\end{array}\right] \oplus\left[\begin{array}{ll}
a_{2} & b_{2} \\
c_{2} & d_{2}
\end{array}\right]=\left[\begin{array}{cccc}
a_{1} & 0 & b_{1} & 0 \\
0 & a_{2} & 0 & b_{2} \\
c_{1} & 0 & d_{1} & 0 \\
0 & c_{2} & 0 & d_{2}
\end{array}\right]
$$

a concatenation of cases 1 and 2 , for example, which we will denote as case $1-2$, thus yields

$$
\left[\begin{array}{cc}
s & 0 \\
0 & s^{-1}
\end{array}\right] \oplus\left[\begin{array}{cc}
\lambda & 1 \\
0 & \lambda
\end{array}\right]=\left[\begin{array}{cccc}
s & 0 & 0 & 0 \\
0 & \lambda & 0 & 1 \\
0 & 0 & s^{-1} & 0 \\
0 & 0 & 0 & \lambda
\end{array}\right] .
$$

In addition to these concatenations, the following four inherently two-dimensional cases arise.

4. A complex quartet of eigenvalues $s \exp (i \theta)$, $s \exp (-i \theta), s^{-1} \exp (-i \theta)$, and $s^{-1} \exp (i \theta)(s \neq \pm 1)$, with four linearly independent eigenvectors; the Jordan matrix reads

$$
\left[\begin{array}{cccc}
s \exp (i \theta) & 0 & 0 & 0 \\
0 & s \exp (-i \theta) & 0 & 0 \\
0 & 0 & s^{-1} \exp (-i \theta) & 0 \\
0 & 0 & 0 & s^{-1} \exp (i \theta)
\end{array}\right] .
$$

This case will be treated in Subsection 5.A. Note that the special unimodular case $s=\exp (i k \pi)$ is considered to belong to case $3-3$.
5. Two identical pairs of unimodular, complex conjugated eigenvalues, $\exp (i \theta)$ and $\exp (-i \theta)$, with only two linearly independent eigenvectors; the Jordan matrix reads

$$
\begin{gathered}
{\left[\begin{array}{cc}
\mathbf{J}_{+}(\exp (i \theta)) & \mathbf{0} \\
\mathbf{0} & \mathbf{J}_{+}(\exp (-i \theta))
\end{array}\right]} \\
\text { or }\left[\begin{array}{cc}
\mathbf{J}_{-}(\exp (i \theta)) & \mathbf{0} \\
\mathbf{0} & \mathbf{J}_{-}(\exp (-i \theta))
\end{array}\right] .
\end{gathered}
$$

This case will be treated in Subsection 5.B.

6. Two identical pairs of real eigenvalues, $s$ and $s^{-1}$ $(s \neq \pm 1)$, with only two linearly independent eigenvectors; the Jordan matrix reads

$$
\left[\begin{array}{cc}
\mathbf{J}_{+}(s) & \mathbf{0} \\
\mathbf{0} & \mathbf{J}_{+}\left(s^{-1}\right)
\end{array}\right] \text { or }\left[\begin{array}{cc}
\mathbf{J}_{-}(s) & \mathbf{0} \\
\mathbf{0} & \mathbf{J}_{-}\left(s^{-1}\right)
\end{array}\right] .
$$

This case will be treated in Subsection 5.C. Again, although the matrices in Eq. (13) resemble the ones in Eq. (12), we will treat the two cases separately. Note that the special unimodular case $s=\exp (i k \pi)$ is considered to belong to case 5 .

7. Four real eigenvalues $\lambda=1$ or $\lambda=-1$, with only one eigenvector; the Jordan matrix reads

$$
\left[\begin{array}{llll}
\lambda & 1 & 0 & 0 \\
0 & \lambda & 1 & 0 \\
0 & 0 & \lambda & 1 \\
0 & 0 & 0 & \lambda
\end{array}\right] \text { or }\left[\begin{array}{cccc}
\lambda & 0 & 0 & 0 \\
-1 & \lambda & 0 & 0 \\
0 & -1 & \lambda & 0 \\
0 & 0 & -1 & \lambda
\end{array}\right] \text {. }
$$

This case will be treated in Subsection 5.D.

An overview of all possible two-dimensional eigenvalue distributions is presented in Table 1 , where the coefficients $a_{1}=a_{3}$ and $a_{2}$ of the characteristic equation $\operatorname{det}(\mathbf{T}-\lambda \mathbf{I})=\lambda^{4}-a_{3} \lambda^{3}+a_{2} \lambda^{2}-a_{1} \lambda+1=0$ are expressed in terms of the eigenvalues for the different two-dimensional cases; the different one-dimensional cases have been extensively treated by Pei and Ding. ${ }^{10}$ We recall that the coefficient $a_{3}$ is the sum of the four entries on the main diagonal of $\mathbf{T}$, while the coefficient $a_{2}$ is the sum of the six $2 \times 2$ determinants that can be built on this diagonal. The coefficient $a_{1}$, which is in general the sum of the four 3 $\times 3$ determinants that can be built on the main diagonal, is equal to $a_{3}$, since both $\lambda$ and $\lambda^{-1}$ are solutions of the characteristic equation, as mentioned before; so, the coefficient $a_{1}$ need not be calculated separately. In terms of the trace and the determinant of the submatrices $\mathbf{A}, \mathbf{B}, \mathbf{C}$, and $\mathbf{D}$, the coefficients read $a_{1}=\operatorname{Tr}(\mathbf{A})+\operatorname{Tr}(\mathbf{D})$ and $a_{2}=\operatorname{det}(\mathbf{A})+\operatorname{det}(\mathbf{D})+\operatorname{Tr}(\mathbf{A}) \operatorname{Tr}(\mathbf{D})-\operatorname{Tr}(\mathbf{B C})$.

The different cases can be represented elegantly in an $a_{1}-a_{2}$ plane (Fig. 1). Two lines (1 and 2) and one parabola (3) are relevant.

1. $a_{2}=2 a_{1}-2$

$$
\begin{array}{ll}
a_{2}>6 & \text { occupied by case } 1-2, \\
a_{2}<-2 & \text { occupied by case } 1-2, \\
-2 \leq a_{2} \leq 6 & \text { occupied by case } 2-3 ;
\end{array}
$$


Table 1. Coefficients for the Different Two-Dimensional Cases ${ }^{a}$

\begin{tabular}{|c|c|c|c|}
\hline Case & Eigenvalues & $a_{1}=a_{3}$ & $a_{2}$ \\
\hline $1-1$ & $s_{1,2}^{ \pm 1}$ & $\left(s_{1}+s_{1}^{-1}\right)+\left(s_{2}+s_{2}^{-1}\right)$ & $2+\left(s_{1}+s_{1}^{-1}\right)\left(s_{2}+s_{2}^{-1}\right)$ \\
\hline $1-2$ & $s^{ \pm 1}, \lambda$ double & $\left(s+s^{-1}\right)+2 \lambda=\left(s+s^{-1}\right) \pm 2$ & $2+2 \lambda\left(s+s^{-1}\right)=2 \pm 2\left(s+s^{-1}\right)$ \\
\hline $1-3$ & $s^{ \pm 1}, \exp ( \pm i \theta)$ & $\left(s+s^{-1}\right)+2 \cos \theta$ & $2+2\left(s+s^{-1}\right) \cos \theta$ \\
\hline 4 & $s^{ \pm 1} \exp ( \pm i \theta)$ & $2\left(s+s^{-1}\right) \cos \theta$ & $2+\left(s^{2}+s^{-2}\right)+2 \cos 2 \theta$ \\
\hline 6 & $s^{ \pm 1}$ double & $2\left(s+s^{-1}\right)$ & $2+\left(s+s^{-1}\right)^{2}$ \\
\hline $2-2$ & $\lambda_{1,2}$ double & $2\left(\lambda_{1}+\lambda_{2}\right)=\left\{\begin{array}{c} \pm 4 \\
0\end{array}\right.$ & $2+4 \lambda_{1} \lambda_{2}=\left\{\begin{array}{c}6 \\
-2\end{array}\right.$ \\
\hline $2-3$ & $\lambda$ double, $\exp ( \pm i \theta)$ & $2(\lambda+\cos \theta)= \pm 2+2 \cos \theta$ & $2+4 \lambda \cos \theta=2 \pm 4 \cos \theta$ \\
\hline $3-3$ & $\exp \left( \pm i \theta_{1,2}\right)$ & $2\left(\cos \theta_{1}+\cos \theta_{2}\right)$ & $2+4 \cos \theta_{1} \cos \theta_{2}$ \\
\hline 5 & $\exp ( \pm i \theta)$ double & $4 \cos \theta$ & $2+4 \cos ^{2} \theta$ \\
\hline 7 & $\lambda$ fourfold & $4 \lambda= \pm 4$ & $2+4 \lambda^{2}=6$ \\
\hline
\end{tabular}

${ }^{a}$ Coefficients $a_{1}=a_{3}$ and $a_{2}$ of the characteristic equation $\lambda^{4}-a_{3} \lambda^{3}+a_{2} \lambda^{2}-a_{1} \lambda+1=0$ for the different two-dimensional cases. The variables $s, s_{1}, s_{2}, \theta, \theta_{1}, \theta_{2}$ are real, $s, s_{1}, s_{2} \neq \pm 1$ and $\lambda, \lambda_{1}, \lambda_{2}= \pm 1$. All cases with unimodular eigenvalues appear in column $a_{2}$ rows 2-2 through 7 .

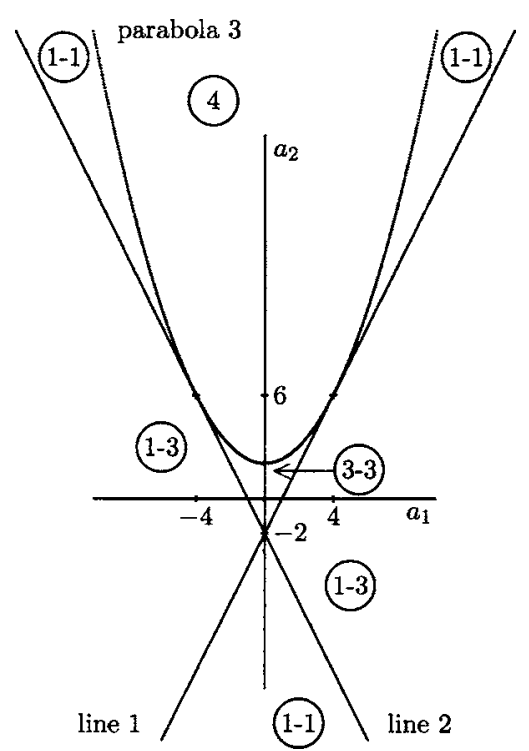

Fig. 1. Different areas for the two-variable cases 1-1, 1-3, 3-3 (boundaries included), and 4 . For the one-variable cases we have case $1-2$, lines 1 and $2\left(a_{2}>6\right.$ or $\left.a_{2}<-2\right)$; case $2-3$, lines 1 and 2 $\left(-2 \leq a_{2} \leq 6\right)$; case 5 , parabola $\left(a_{2} \leq 6\right)$; case 6 , parabola $\left(a_{2}>6\right)$. For the isolated points we have case 2-2, $\left(a_{1}, a_{2}\right)=( \pm 4,6)$ or $\left(a_{1}, a_{2}\right)=(0,-2)$ and case $7,\left(a_{1}, a_{2}\right)=( \pm 4,6)$. Cases $2-2,2-3,3-3,5$, and 7 correspond to unimodular eigenvalues, while the other cases have two (cases 1-2 and 1-3) or four (cases 1-1, 4, and 6) nonunimodular eigenvalues.

2. $a_{2}=-2 a_{1}-2$ :

$$
\begin{array}{ll}
a_{2}>6 & \text { occupied by case } 1-2, \\
a_{2}<-2 & \text { occupied by case } 1-2, \\
-2 \leq a_{2} \leq 6 & \text { occupied by case } 2-3 ;
\end{array}
$$

3. $a_{2}=2+a_{1}^{2} / 4$ :

$$
\begin{array}{ll}
a_{2}>6 & \text { occupied by case } 6 \\
2 \leq a_{2} \leq 6 & \text { occupied by case } 5
\end{array}
$$

together with the three points

$$
\begin{array}{ll}
\left(a_{1}, a_{2}\right)=(4,6) & \text { occupied by cases } 2-2 \text { and } 7, \\
\left(a_{1}, a_{2}\right)=(-4,6) & \text { occupied by cases } 2-2 \text { and } 7, \\
\left(a_{1}, a_{2}\right)=(0,-2) & \text { occupied by case } 2-2 .
\end{array}
$$

The area between the two lines and the parabola, the boundaries included, is occupied by case 3-3 and contains the unimodular eigenvalues. The areas between line 1 and the parabola $\left(a_{2}>6\right)$, between line 2 and the parabola $\left(a_{2}>6\right)$, and between lines 1 and $2\left(a_{2}<-2\right)$, the boundaries themselves not included, are occupied by case 1-1. The area above the parabola $\left(a_{2}>2\right)$, the parabola itself not included, is occupied by case 4 . The two remaining areas, the lines 1 and 2 themselves not included, are occupied by case 1-3. We remark that only the two-variable cases 1-1, 1-3, 3-3, and 4 occupy two-dimensional areas, as can be expected; the other cases occupy only lines (the one-variable cases 1-2, 2-3, 5, and 6) or isolated points (cases 2-2 and 7).

The problem with the common Jordan matrix associated to a real symplectic matrix is that it does not automatically preserve the properties of realness [Eqs. (8), (11), and (12)] and symplecticity [Eq. (14)]. Instead of the Jordan matrix, we therefore look for other matrices in these four cases: matrices with a minimum number of parameters, which do preserve these properties. Since we only deal with $D=1$ and $D=2$, we can easily formulate these matrices. For higher-dimensional real symplectic matrices, we refer to the paper by Lin et al. ${ }^{19}$ in particular Theorem 41. We remark that Lin et al. ${ }^{19}$ treat both symplectic and Hamiltonian matrices. In view of this, the reader may also benefit from a comparison of our results for real symplectic matrices to those for Hamiltonian matrices as described in Ref. 6 (Chapter 12, Hamiltonian orbits). Since Hamiltonian matrices are the logarithms of symplectic matrices, our Fig. 1, for example, may be directly connected to Figs. 12.3 and 12.4 in Ref. 6.

\section{NUCLEI OF REAL SYMPLECTIC MATRICES: ONE-DIMENSIONAL CASE}

We will now present nuclei for the three cases that may occur for $D=1$. 
A. Magnifier $\mathcal{M}(s)$ : Case 1 [Eq. (6)]

The magnifier $\mathcal{M}(s)$, with ray transformation matrix

$$
\mathbf{T}_{\mathcal{M}}(s)=\Lambda_{\mathcal{M}}(s)=\left[\begin{array}{cc}
s & 0 \\
0 & s^{-1}
\end{array}\right],
$$

is an obvious nucleus for the case of a pair of two (generally different) real eigenvalues: $s$ and $s^{-1}$. In particular, we have the unity operator $\mathbf{T}_{\mathcal{M}}(1)=\mathbf{I}$ when $s=1$ and the coordinate reverter $\mathbf{T}_{\mathcal{M}}(-1)=-\mathbf{I}$ when $s=-1$. In general, we will restrict ourselves to real eigenvalues $s$ that are positive; in the case of negative real eigenvalues we simply add an additional coordinate reverter -I to the nucleus.

Another possible nucleus in this class would be the same magnifier $\mathcal{M}(s)$ embedded in between a fractional Fourier transformer $\mathcal{F}(-\pi / 4 ; w)$ and its inverse. The corresponding ray transformation matrix of this nucleus reads, with $s=\exp (\sigma)>0$,

$$
\begin{aligned}
& \mathbf{T}_{\mathcal{F}}(-\pi / 4 ; w) \mathbf{T}_{\mathcal{M}}(\exp \sigma) \mathbf{T}_{\mathcal{F}}(\pi / 4 ; w) \\
& \quad=\left[\begin{array}{cc}
\cosh \sigma & w^{2} \sinh \sigma \\
w^{-2} \sinh \sigma & \cosh \sigma
\end{array}\right] \equiv \mathbf{T}_{\mathcal{H}}(\sigma ; w),
\end{aligned}
$$

and is associated to a so-called hyperbolic expander (see Ref. 6, p. 183, Example: Hyperbolic expanders). Any diagonalizable $A B C D$ system from this class (i.e., with $A D$ $-B C=1$ and $|A+D| \geq 2$ ) can be decomposed as a hyperbolic expander $\mathcal{H}(\sigma ; w)$ embedded in between two lenses with opposite focal lengths. For the $A B C D$ matrix we thus get

$$
\begin{aligned}
\mathbf{T}= & {\left[\begin{array}{ll}
A & B \\
C & D
\end{array}\right]=\frac{A+D}{|A+D|}\left[\begin{array}{cc}
1 & 0 \\
-g & 1
\end{array}\right] } \\
& \times\left[\begin{array}{cc}
\cosh \sigma & w^{2} \sinh \sigma \\
w^{-2} \sinh \sigma & \cosh \sigma
\end{array}\right]\left[\begin{array}{ll}
1 & 0 \\
g & 1
\end{array}\right],
\end{aligned}
$$

where $\sigma$ and $w^{2}$ follow from $|A+D|=2 \cosh \sigma$ and $B(A$ $+D) /|A+D|=w^{2} \sinh \sigma$, and $g=1 / \lambda_{0} f$ follows from $A-D$ $=2 g B$. Note that the expression $(A+D) /|A+D|$ equals either +1 or -1 for the two possible cases $A+D \geq 2$ and $A$ $+D \leq-2$, respectively.

\section{B. Lens $\mathcal{L}(f)$ and Free Space $S(z)$ : Case 2 [Eq. (7)]}

The lens $\mathcal{L}(f)$ and a section of free space $S(z)$ with ray transformation matrices $\mathbf{T}_{\mathcal{L}}(f)$ and $\mathbf{T}_{\mathcal{S}}(z)$ [Eqs. (4)], respectively, are obvious nuclei for the case of two identical eigenvalues $\lambda=1$ with only one eigenvector. The case of two negative eigenvalues $\lambda=-1$ can easily be dealt with, again, by means of an additional coordinate reverter.

The case of a double eigenvalue $\lambda= \pm 1$ corresponds to the case where the diagonal entries of the $2 \times 2 A B C D$ matrix satisfy the condition $A+D= \pm 2$, and which has been extensively treated by Pei and Ding (Ref. 10, Section IV). If $A=D= \pm 1$, the system is obviously either a unity operator (for $B=C=0$ ), or a section of free space (for $B$ $=\lambda_{0} z, C=0$ ), or a lens (for $B=0, C=-1 / \lambda_{0} f$ ), possibly with an additional coordinate reversion (if $\lambda=-1$ ). For $A \neq D$ (and consequently $B C=A D-1<0$ ), the system can always be presented as a lens with $g=1 / \lambda_{0} f$ (or as a section of free space; see Ref. 10, Sections IV.D and IV.E) embedded in between an abcd system (with $a d-b c=1$ ) and its inverse, and possibly with an additional coordinate reversion. In detail we have

$$
\begin{aligned}
\mathbf{T} & =\left[\begin{array}{ll}
A & B \\
C & D
\end{array}\right]=\left[\begin{array}{ll}
a & b \\
c & d
\end{array}\right]( \pm 1)\left[\begin{array}{cc}
1 & 0 \\
-g & 1
\end{array}\right]\left[\begin{array}{cc}
d & -b \\
-c & a
\end{array}\right] \\
& =( \pm 1)\left[\begin{array}{cc}
1-b d g & b^{2} g \\
-d^{2} g & 1+b d g
\end{array}\right]
\end{aligned}
$$

where, for example, $a$ and $d \neq 0$ can be chosen arbitrarily, $b=[2 B /(D-A)] d, c=(a d-1) / b$, and $g=\left(1-\lambda^{-1} A\right) / b d$. Note that the two-dimensional column vector $[b, d]^{t}=\mathbf{q}_{1}$ is the (only) eigenvector (with $\mathbf{T} \mathbf{q}_{1}=\lambda \mathbf{q}_{1}$ ), whereas $[a, c]^{t}=\mathbf{q}_{2}$ is a generalized eigenvector [with $\mathbf{T}\left(\mathbf{q}_{2}+g \mathbf{q}_{1}\right)=\lambda \mathbf{q}_{2}$ ]. To convert the lens into free space, or vice versa, if necessary, we simply embed it into a Fourier transformer and its inverse [see Eq. (5)] and get

$$
\left[\begin{array}{cc}
0 & w^{2} \\
-w^{-2} & 0
\end{array}\right]\left[\begin{array}{cc}
1 & 0 \\
-g & 1
\end{array}\right]\left[\begin{array}{cc}
0 & -w^{2} \\
w^{-2} & 0
\end{array}\right]=\left[\begin{array}{cc}
1 & g w^{4} \\
0 & 1
\end{array}\right] \text {. }
$$

Note that this relation can be used to realize a section of free space with a negative distance $z<0$, using a divergent lens $\left(g=1 / \lambda_{\mathrm{o}} f<0\right)$.

For completeness, we also give the decomposition in the form where a section of free space with $h=\lambda_{0} z$ is embedded in between an abcd system and its inverse:

$$
\begin{aligned}
\mathbf{T} & =\left[\begin{array}{ll}
A & B \\
C & D
\end{array}\right]=\left[\begin{array}{ll}
a & b \\
c & d
\end{array}\right]( \pm 1)\left[\begin{array}{cc}
1 & h \\
0 & 1
\end{array}\right]\left[\begin{array}{cc}
d & -b \\
-c & a
\end{array}\right] \\
& =( \pm 1)\left[\begin{array}{cc}
1-a c h & a^{2} h \\
-c^{2} h & 1+a c h
\end{array}\right],
\end{aligned}
$$

where now, for example, $a \neq 0$ and $d$ can be chosen arbitrarily, $\quad c=[(D-A) / 2 B] a, b=(a d-1) / c, \quad$ and $\quad h=(1$ $\left.-\lambda^{-1} A\right) / a c$. Note that the two-dimensional column vector $[a, c]^{t}=\mathbf{q}_{1}$ is the (only) eigenvector (with $\mathbf{T} \mathbf{q}_{1}=\lambda \mathbf{q}_{1}$ ), whereas $[b, d]^{t}=\mathbf{q}_{2}$ is the generalized eigenvector [with $\left.\mathbf{T}\left(\mathbf{q}_{2}-h \mathbf{q}_{1}\right)=\lambda \mathbf{q}_{2}\right]$.

\section{Fractional Fourier Transformer $\mathcal{F}(\theta ; w)$ : Case 3}

[Eq. (8)]

The fractional Fourier transformer $\mathcal{F}(\theta ; w)$, with ray transformation matrix $\mathbf{T}_{\mathcal{F}}(\theta ; w)$ [Eq. (5)]

$$
\begin{aligned}
\mathbf{T}_{\mathcal{F}}(\theta ; w)= & \frac{1}{\sqrt{2}}\left[\begin{array}{cc}
1 & i w^{2} \\
i w^{-2} & 1
\end{array}\right] \\
& \times\left[\begin{array}{cc}
\exp (i \theta) & 0 \\
0 & \exp (-i \theta)
\end{array}\right] \sqrt{2}\left[\begin{array}{cc}
1 & i w^{2} \\
i w^{-2} & 1
\end{array}\right]^{-1} \\
\equiv & \mathbf{Q}_{\mathcal{F}}(w) \boldsymbol{\Lambda}_{\mathcal{F}}(\theta) \mathbf{Q}_{\mathcal{F}}^{-1}(w)
\end{aligned}
$$

is an obvious nucleus for the case of a pair of two (generally different) complex conjugated unimodular eigenvalues $\exp (i \theta)$ and $\exp (-i \theta)$. Note that the eigenvalue matrix $\boldsymbol{\Lambda}_{\mathcal{F}}(\theta)$ [Eq. (8)] and the eigenvector matrix $\mathbf{Q}_{\mathcal{F}}(w)$ are complex, and that the eigenvalue matrix itself thus cannot act as the ray transformation matrix of a proper nucleus.

For this class we can decompose the diagonalizable $A B C D$ matrix (with $A D-B C=1$ and $|A+D| \leq 2$ ) as 


$$
\begin{aligned}
\mathbf{T} & =\left[\begin{array}{ll}
A & B \\
C & D
\end{array}\right] \\
& =\left[\begin{array}{cc}
1 & 0 \\
-g & 1
\end{array}\right]\left[\begin{array}{cc}
\cos \theta & w^{2} \sin \theta \\
-w^{-2} \sin \theta & \cos \theta
\end{array}\right]\left[\begin{array}{ll}
1 & 0 \\
g & 1
\end{array}\right],
\end{aligned}
$$

where $\theta$ and $w^{2}$ follows from $A+D=2 \cos \theta$ and $B$ $=w^{2} \sin \theta$, and $g=1 / \lambda_{0} f$ follows again from $A-D=2 g B$. Note the resemblance to the case of the real eigenvalues [Eq. (17)]; the two lenses now embed a fractional Fourier transformer instead of a hyperbolic expander. The $\mathcal{M}$ class (Subsection 4.A) and the $\mathcal{F}$ class (Subsection 4.C) touch each other for $A=D=1$ and $B=C=0$, in which case the (double) eigenvalues are either +1 or -1 , and thus $\sin \theta=\sigma=0$.

\section{NUCLEI OF REAL SYMPLECTIC MATRICES: TWO-DIMENSIONAL CASE}

A class of separable nuclei for two-dimensional optical systems, $D=2$, can be constructed by concatenation of one-dimensional nuclei. We thus get, for example, separable nuclei such as a separable magnifier $\mathcal{M}\left(s_{x}, s_{y}\right)$ [Eq. (15)], an anamorphic separable lens $\mathcal{L}\left(f_{x}, f_{y}\right)$ [Eqs. (4)], and a separable fractional Fourier transformer $\mathcal{F}\left(\theta_{x}, \theta_{y} ; w_{x}, w_{y}\right)$ [Eq. (5)], with ray transformation matrices

$$
\begin{aligned}
\mathbf{T}_{\mathcal{M}}\left(s_{x}, s_{y}\right) & =\mathbf{T}_{\mathcal{M}}\left(s_{x}\right) \oplus \mathbf{T}_{\mathcal{M}}\left(s_{y}\right), \\
\mathbf{T}_{\mathcal{L}}\left(f_{x}, f_{y}\right) & =\mathbf{T}_{\mathcal{L}}\left(f_{x}\right) \oplus \mathbf{T}_{\mathcal{L}}\left(f_{y}\right), \\
\mathbf{T}_{\mathcal{F}}\left(\theta_{x}, \theta_{y} ; w_{x}, w_{y}\right) & =\mathbf{T}_{\mathcal{F}}\left(\theta_{x} ; w_{x}\right) \oplus \mathbf{T}_{\mathcal{F}}\left(\theta_{y} ; w_{y}\right),
\end{aligned}
$$

which correspond to cases 1-1, 2-2, and 3-3, respectively. Nuclei from different classes can be concatenated as well. We thus get, for example,

$$
\begin{gathered}
\mathbf{T}_{\mathcal{M}}\left(s_{x}\right) \oplus \mathbf{T}_{\mathcal{L}}\left(f_{y}\right), \\
\mathbf{T}_{\mathcal{M}}\left(s_{x}\right) \oplus \mathbf{T}_{\mathcal{F}}\left(\theta_{y} ; w_{y}\right),
\end{gathered}
$$

$$
\mathbf{T}_{\mathcal{L}}\left(f_{x}\right) \oplus \mathbf{T}_{\mathcal{F}}\left(\theta_{y} ; w_{y}\right),
$$

for the three classes 1-2, 1-3, and 2-3, respectively. By combining a lens in the $x$ dimension with a fractional Fourier transformer in the $y$ dimension, we get a nucleus with the ray transformation matrix $\mathbf{T}_{\mathcal{L}}\left(f_{x}\right) \oplus \mathbf{T}_{\mathcal{F}}\left(\theta_{y} ; w_{y}\right)$ :

$$
\left[\begin{array}{cccc}
1 & 0 & 0 & 0 \\
0 & \cos \theta_{y} & 0 & w_{y}^{2} \sin \theta_{y} \\
-1 / \lambda_{\circ} f_{x} & 0 & 1 & 0 \\
0 & -w_{y}^{-2} \sin \theta_{y} & 0 & \cos \theta_{y}
\end{array}\right] .
$$

The four remaining two-dimensional nuclei, corresponding to the cases $4,5,6$, and 7 , are inherently twodimensional: one nucleus (case 4) with a still sufficient number of four linearly independent eigenvectors, and the other three nuclei (cases 5, 6, and 7) with fewer eigenvectors. We will be able to choose all four remaining nuclei such that they will perform operations between conjugate planes, like a magnifier and a lens do [with $\mathbf{B}=\mathbf{0}$ ], and to realize these nuclei in practice we need combinations of a rotator or a shearer with an isotropic magnifier or an isotropic lens. Note that the rotator and the shearer are inherently two-dimensional, whereas the isotropic magnifier and the isotropic lens are concatenations of their one-dimensional versions.

\section{A. Rotator-Magnifier Combination $\mathcal{M}(s, s) \mathcal{R}(\theta)$ : Case 4} [Eq. (11)]

There is one obvious case in the realm of diagonalizable real symplectic matrices that can only occur for $D>1$, viz., the one in which we have a full complex quartet of eigenvalues: $\lambda, \lambda^{*}, 1 / \lambda$, and $1 / \lambda^{*}$, with $\lambda$ not unimodular and not real. A possible nucleus for this class, with eigenvalues $s \exp ( \pm i \theta)$ and $s^{-1} \exp ( \pm i \theta)$, is the system with ray transformation matrix

$$
\mathbf{T}_{\mathcal{M R}}(\theta, s)=\left[\begin{array}{cc}
s \mathbf{R}(\theta) & \mathbf{0} \\
\mathbf{0} & s^{-1} \mathbf{R}(\theta)
\end{array}\right],
$$

where the $2 \times 2$ matrix $\mathbf{R}(\theta)$ is the rotation matrix

$$
\mathbf{R}(\theta)=\left[\begin{array}{cc}
\cos \theta & \sin \theta \\
-\sin \theta & \cos \theta
\end{array}\right] .
$$

In detail we have

$$
\begin{aligned}
& \mathbf{T}_{\mathcal{M R}}(\theta, s)=\left[\begin{array}{cccc}
s \cos \theta & s \sin \theta & 0 & 0 \\
-s \sin \theta & s \cos \theta & 0 & 0 \\
0 & 0 & s^{-1} \cos \theta & s^{-1} \sin \theta \\
0 & 0 & -s^{-1} \sin \theta & s^{-1} \cos \theta
\end{array}\right], \\
& =\frac{1}{\sqrt{2}}\left[\begin{array}{cccc}
1 & i & 0 & 0 \\
i & 1 & 0 & 0 \\
0 & 0 & 1 & -i \\
0 & 0 & -i & 1
\end{array}\right]\left[\begin{array}{cccc}
s \exp (i \theta) & 0 & 0 & 0 \\
0 & s \exp (-i \theta) & 0 & 0 \\
0 & 0 & s^{-1} \exp (-i \theta) & 0 \\
0 & 0 & 0 & s^{-1} \exp (i \theta)
\end{array}\right] \sqrt{2}\left[\begin{array}{cccc}
1 & i & 0 & 0 \\
i & 1 & 0 & 0 \\
0 & 0 & 1 \\
0 & 0 & -i & 1
\end{array}\right]^{-1}
\end{aligned}
$$


Note again that the eigenvalue matrix [Eq. (11)] and the eigenvector matrix are complex, and that the eigenvalue matrix thus cannot act as the ray transformation matrix of a proper nucleus.

The matrix $\mathbf{T}_{\mathcal{M R}}(\theta, s)$ is clearly the ray transformation matrix of a combination of a rotator $\mathcal{R}(\theta)$ [also called image gyrator; see Ref. 20, Eq. (49)] with ray transformation matrix

$$
\mathbf{T}_{\mathcal{R}}(\theta)=\left[\begin{array}{cc}
\mathbf{R}(\theta) & \mathbf{0} \\
\mathbf{0} & \mathbf{R}(\theta)
\end{array}\right]
$$

and an isotropic magnifier $\mathcal{M}(s, s)$ [Eqs. (15) and (23)]:

$$
\mathbf{T}_{\mathcal{M R}}(\theta, s)=\mathbf{T}_{\mathcal{M}}(s, s) \mathbf{T}_{\mathcal{R}}(\theta)=\mathbf{T}_{\mathcal{R}}(\theta) \mathbf{T}_{\mathcal{M}}(s, s) .
$$

Note that the matrices $\mathbf{T}_{\mathcal{R}}(\theta)$ and $\mathbf{T}_{\mathcal{M}}(s, s)$ in the latter expression commute and that the order in the cascade does not matter. Note also that $\mathbf{T}_{\mathcal{M R}}(\theta, s)$ reduces to a rotator $\mathbf{T}_{\mathcal{R}}(\theta)$ for $s=1$, and to an isotropic magnifier $\mathbf{T}_{\mathcal{M}}(s, s)$ for $\theta=0$.

From the general input-output relationship

$$
f_{o}(\mathbf{r})=f_{i}\left(\mathbf{A}^{-1} \mathbf{r}\right) \exp \left(i \pi \mathbf{r}^{t} \mathbf{C A}^{-1} \mathbf{r}\right) / \sqrt{|\operatorname{det} \mathbf{A}|},
$$

which holds for a linear canonical transformation $f_{i}(\mathbf{r})$ $\rightarrow f_{o}(\mathbf{r})$ in the special case that $\mathbf{B}=\mathbf{0}$, we immediately derive the input-output relation for the rotator-magnifier combination [with $\mathbf{A}=s \mathbf{R}(\theta)$ and $\mathbf{C}=\mathbf{0}$ ] as

$$
s f_{o}(s x, s y)=f_{i}(x \cos \theta-y \sin \theta, x \sin \theta+y \cos \theta),
$$

or in polar coordinates (with $x=r \cos \varphi$ and $y=r \sin \varphi$ ):

$$
s f_{o}(s r, \varphi)=f_{i}(r, \varphi+\theta) \text {. }
$$

As we converted, in Subsection 4.A, the magnifier $\mathcal{M}(\exp \sigma)$ into a hyperbolic expander $\mathcal{H}(\sigma ; w)$ by embedding it in between a fractional Fourier transformer with fractional angle $-\pi / 4$ and its inverse [Eq. (16)], we can do the same here to find an alternative nucleus: a combination of an isotropic hyperbolic expander and a rotator$\mathcal{H}(\sigma, \sigma ; w, w) \mathcal{R}(\theta)$.

\section{B. Rotator-Lens Combination $\mathcal{L}(f, f) \mathcal{R}(\theta)$ : Case 5}

[Eq. (12)]

From the nondiagonalizable matrices, let us first consider the case of a double pair of complex conjugated unimodular eigenvalues. A possible nucleus for this class, with double eigenvalues $\exp ( \pm i \theta)$, is the system with ray transformation matrix

$$
\mathbf{T}_{\mathcal{L R}}(\theta ; f)=\left[\begin{array}{cc}
\mathbf{R}(\theta) & \mathbf{0} \\
-\left(1 / \lambda_{\mathrm{o}} f\right) \mathbf{R}(\theta) & \mathbf{R}(\theta)
\end{array}\right]
$$

which is a (commuting) combination of a rotator $\mathcal{R}(\theta)$ [Eq. (33)] and an isotropic lens $\mathcal{L}(f, f)$ [Eqs. (4) and (24)]:

$$
\mathbf{T}_{\mathcal{L R}}(\theta ; f)=\mathbf{T}_{\mathcal{L}}(f, f) \mathbf{T}_{\mathcal{R}}(\theta)=\mathbf{T}_{\mathcal{R}}(\theta) \mathbf{T}_{\mathcal{L}}(f, f) .
$$

The input-output relation of this system [with $\mathbf{A}=\mathbf{R}(\theta)$ and $\left.\mathbf{C}=-\left(1 / \lambda_{\circ} f\right) \mathbf{R}(\theta)\right]$ reads

$$
\begin{aligned}
f_{o}(x, y)= & f_{i}(x \cos \theta-y \sin \theta, x \sin \theta+y \cos \theta) \\
& \times \exp \left[-i \pi\left(1 / \lambda_{\mathrm{o}} f\right)\left(x^{2}+y^{2}\right)\right],
\end{aligned}
$$

or in polar coordinates again

$$
f_{o}(r, \varphi)=f_{i}(r, \varphi+\theta) \exp \left[-i \pi\left(1 / \lambda_{o} f\right) r^{2}\right] .
$$

Just as a lens and a section of free space are each others' Fourier transforms [Eq. (19)], we can also find an alternative nucleus $\mathcal{F}(\pi / 2 ; w) \mathcal{L}(f, f) \mathcal{R}(\theta) \mathcal{F}(-\pi / 2 ; w)$ $=S\left(w^{4} / \lambda_{0} f\right) \mathcal{R}(\theta)$ by embedding the rotator-lens combination in between a Fourier transformer and its inverse.

\section{Shearer-Magnifier Combination $\mathcal{M}(s, s) Z$ : Case 6} [Eq. (13)]

Let us now consider the case of a double pair of real eigenvalues; as mentioned before, we will restrict ourselves to the case of positive real eigenvalues. A possible nucleus for this class, with double eigenvalues $s$ and $s^{-1}$, is the system with ray transformation matrix

$$
\mathbf{T}_{\mathcal{M Z}}(s)=\left[\begin{array}{cc}
s \mathbf{J}_{+} & \mathbf{0} \\
\mathbf{0} & s^{-1} \mathbf{J}_{-}
\end{array}\right]=\left[\begin{array}{cc}
s \mathbf{I} & \mathbf{0} \\
\mathbf{0} & s^{-1} \mathbf{I}
\end{array}\right]\left[\begin{array}{cc}
\mathbf{J}_{+} & \mathbf{0} \\
\mathbf{0} & \mathbf{J}_{-}
\end{array}\right],
$$

which is a (commuting) combination of a shearer $\mathcal{Z}$ with ray transformation matrix

$$
\mathbf{T}_{\mathcal{Z}}=\left[\begin{array}{cc}
\mathbf{J}_{+} & \mathbf{0} \\
\mathbf{0} & \mathbf{J}_{-}
\end{array}\right]
$$

and an isotropic magnifier $\mathcal{M}(s, s)$ :

$$
\mathbf{T}_{\mathcal{M Z}}(s)=\mathbf{T}_{\mathcal{M}}(s, s) \mathbf{T}_{\mathcal{Z}}=\mathbf{T}_{\mathcal{Z}} \mathbf{T}_{\mathcal{M}}(s, s) \text {. }
$$

The input-output relation of this system [with $\mathbf{A}$ $=s \mathbf{J}_{+}$and $\left.\mathbf{C}=\mathbf{0}\right]$ reads

$$
s f_{o}(s x, s y)=f_{i}(x-y, y),
$$

which represents, apart from a magnification with $s$, a simple shearing of the $x$ coordinate.

Using the Iwasawa decomposition ${ }^{21}$ (see also Ref. 6, Sections 9.5 and 10.2), we can express the shearer as a cascade of a magnifier $\mathcal{M}$ and a rotator $\mathcal{R}$ :

$$
\begin{aligned}
\mathbf{T}_{\mathcal{Z}}= & {\left[\begin{array}{cc}
\mathbf{J}_{+} & \mathbf{0} \\
\mathbf{0} & \mathbf{J}_{-}
\end{array}\right]=\left[\begin{array}{cc}
\left(\mathbf{J}_{+} \mathbf{J}_{+}^{t}\right)^{1 / 2} & \mathbf{0} \\
\mathbf{0} & \left(\mathbf{J}_{+} \mathbf{J}_{+}^{t}\right)^{-1 / 2}
\end{array}\right] } \\
& \times\left[\begin{array}{cc}
\left(\mathbf{J}_{+} \mathbf{J}_{+}^{t}\right)^{-1 / 2} \mathbf{J}_{+} & \mathbf{0} \\
\mathbf{0} & \left(\mathbf{J}_{+} \mathbf{J}_{+}^{t}\right)^{-1 / 2} \mathbf{J}_{+}
\end{array}\right] .
\end{aligned}
$$

With $\phi$ the golden ratio, $\phi=(\sqrt{5}+1) / 2=2 /(\sqrt{5}-1)$ satisfying the quadratic equation $\phi^{2}-\phi-1=0$, the eigenvalues of $\mathbf{J}_{+} \mathbf{J}_{+}^{t}$ are $\phi^{2}$ and $\phi^{-2}$, and we can write the similarity relation

$$
\mathbf{J}_{+} \mathbf{J}_{+}^{t}=\left[\begin{array}{cc}
\phi & -1 \\
1 & \phi
\end{array}\right]\left[\begin{array}{cc}
\phi^{2} & 0 \\
0 & \phi^{-2}
\end{array}\right]\left[\begin{array}{cc}
\phi & -1 \\
1 & \phi
\end{array}\right]^{-1} .
$$

We thus find

$$
\begin{aligned}
\left(\mathbf{J}_{+} \mathbf{J}_{+}^{t}\right)^{1 / 2} & =\left[\begin{array}{cc}
\phi & -1 \\
1 & \phi
\end{array}\right]\left[\begin{array}{cc}
\phi & 0 \\
0 & \phi^{-1}
\end{array}\right]\left[\begin{array}{cc}
\phi & -1 \\
1 & \phi
\end{array}\right]^{-1} \\
& =\frac{1}{\sqrt{5}}\left[\begin{array}{lr}
3 & 1 \\
1 & 2
\end{array}\right] \equiv\left[\begin{array}{cc}
s_{11} & s_{12} \\
s_{12} & s_{22}
\end{array}\right]
\end{aligned}
$$




$$
\begin{aligned}
\left(\mathbf{J}_{+} \mathbf{J}_{+}^{t}\right)^{-1 / 2} \mathbf{J}_{+} & =\frac{1}{\sqrt{5}}\left[\begin{array}{ll}
3 & 1 \\
1 & 2
\end{array}\right]^{-1}\left[\begin{array}{ll}
1 & 1 \\
0 & 1
\end{array}\right]=\frac{1}{\sqrt{5}}\left[\begin{array}{cc}
2 & 1 \\
-1 & 2
\end{array}\right] \\
& \equiv \mathbf{R}(\alpha),
\end{aligned}
$$

we conclude that a shearer can be realized as an anamorphic magnifier preceded by a rotator whose rotation angle $\alpha$ is determined by $\cos \alpha=2 / \sqrt{5}$ and $\sin \alpha=1 / \sqrt{5}$. The anamorphic magnifier itself can be represented as a separable magnifier $\mathcal{M}\left(\phi, \phi^{-1}\right)$ embedded in between a rotator $\mathcal{R}(\beta)$ and its inverse, $\mathcal{R}(\beta) \mathcal{M}\left(\phi, \phi^{-1}\right) \mathcal{R}(-\beta)$, where $\beta=$ $-\operatorname{arccot} \phi$. The two adjacent rotators can, of course, be combined, so that the shearer can finally be realized as a separable magnifier embedded in between two rotators:

$$
\mathcal{Z}=\mathcal{R}(-\operatorname{arccot} \phi) \mathcal{M}\left(\phi, \phi^{-1}\right) \mathcal{R}(\operatorname{arccot} \phi+\operatorname{arccot} 2) .
$$

\section{Shearer-Lens Combination $\mathcal{L}(f, f) \mathcal{Z}$ : Case 7 [Eq. (14)]}

We finally consider the case of a fourfold eigenvalue $\lambda=1$; for convenience we restrict ourselves again to the case $\lambda$ $=1$, while the case $\lambda=-1$ would simply require an additional coordinate reverter -I. A possible nucleus for this class is the system with ray transformation matrix

$$
\begin{aligned}
\mathbf{T}_{\mathcal{L Z}}(f) & =\left[\begin{array}{cc}
\mathbf{J}_{+} & \mathbf{0} \\
-\left(1 / \lambda_{\mathrm{o}} f\right) \mathbf{J}_{+} & \mathbf{J}_{-}
\end{array}\right] \\
& =\left[\begin{array}{cc}
\mathbf{I} & \mathbf{0} \\
-\left(1 / \lambda_{\mathrm{o}} f\right) \mathbf{I} & \mathbf{I}
\end{array}\right]\left[\begin{array}{cc}
\mathbf{J}_{+} & \mathbf{0} \\
\mathbf{0} & \mathbf{J}_{-}
\end{array}\right],
\end{aligned}
$$

which is a (not commuting) combination of a shearer $\mathcal{Z}$ with an isotropic lens $\mathcal{L}(f, f)$ :

$$
\mathbf{T}_{\mathcal{L Z}}=\mathbf{T}_{\mathcal{L}}(f, f) \mathbf{T}_{\mathcal{Z}}
$$

The input-output relation of this system [with $\mathbf{A}=\mathbf{J}_{+}$and $\left.\mathbf{C}=-\left(1 / \lambda_{0} f\right) \mathbf{J}_{+}\right]$reads

$$
f_{o}(x, y)=f_{i}(x-y, y) \exp \left[-i \pi\left(1 / \lambda_{\mathrm{o}} f\right)\left(x^{2}+y^{2}\right)\right] .
$$

Just as a lens and a section of free space are each others' Fourier transforms [Eq. (19)], we can also find an alternative nucleus $\mathcal{F}(\pi / 2 ; w) \mathcal{L}(f, f) Z \mathcal{F}(-\pi / 2 ; w)=\mathcal{S}\left(w^{4} / \lambda_{\mathrm{o}} f\right) Z^{\prime}$ by embedding the shearer-lens combination in between a Fourier transformer and its inverse. The ray transformation matrices of the shearers $\mathcal{Z}^{\prime}$ and $\mathcal{Z}$ differ only in the positions of the Jordan blocks $\mathbf{J}_{+}$and $\mathbf{J}_{-}$[Eq. (43)], and we have $\mathbf{T}_{\mathcal{Z}}^{-1}=\mathbf{T}_{\mathcal{Z}}^{t}$.

The results of Sections 4 and 5 have been combined in Table 2, where for each of the seven classes the corresponding nucleus can be extracted. The nucleus for the class presented in Subsection 5.D, for example, with four eigenvalues equal to 1 and with only one eigenvector, consists of a combination of a shearer $\mathcal{Z}$ followed by a lens $\mathcal{L}$ (or a section of free space $\mathcal{S}$ ), and the two subsystems do not commute.

\section{EIGENFUNCTIONS OF FIRST-ORDER OPTICAL SYSTEMS}

Any lossless first-order optical system is associated to a linear canonical integral transformation, $f_{i}(\mathbf{r}) \rightarrow f_{o}(\mathbf{r})$ $=\mathcal{T}^{\mathbf{T}}\left[f_{i}(\cdot)\right](\mathbf{r})$, parameterized by the ray transformation matrix $\mathbf{T}$ of the first-order optical system. If the submatrix $\mathbf{B}$ in the ray transformation matrix is not singular, the canonical transformation can be represented by Collins' integral ${ }^{7}$ :

$$
\begin{aligned}
f_{o}\left(\mathbf{r}_{o}\right)= & \mathcal{T}^{\mathbf{T}}\left[f_{i}\left(\mathbf{r}_{i}\right)\right]\left(\mathbf{r}_{o}\right)=\frac{1}{\sqrt{\operatorname{det} i \mathbf{B}}} \int_{-\infty}^{\infty} f_{i}\left(\mathbf{r}_{i}\right) \\
& \times \exp \left[i \pi\left(\mathbf{r}_{i}^{t} \mathbf{B}^{-1} \mathbf{A} \mathbf{r}_{i}-2 \mathbf{r}_{i}^{t} \mathbf{B}^{-1} \mathbf{r}_{o}+\mathbf{r}_{o}^{t} \mathbf{D} \mathbf{B}^{-1} \mathbf{r}_{o}\right)\right] \mathrm{d} \mathbf{r}_{i} .
\end{aligned}
$$

In the limit $\mathbf{B} \rightarrow \mathbf{0}$ (and hence $\mathbf{A}^{-1}=\mathbf{D}^{t}$ exists), the transformation reduces to the form given in Eq. (35). The case that $\mathbf{B}$ is singular but $\mathbf{B} \neq \mathbf{0}$ can be treated as well, at least for $D=2$. $^{22}$

Knowledge of a nucleus $\mathbf{T}_{n}$ may be helpful in finding eigenfunctions of the corresponding class of first-order optical systems, decomposable as $\mathbf{M} \mathbf{T}_{n} \mathbf{M}^{-1}$. Indeed, if $\Phi(\mathbf{r})$ is a known eigenfunction of the nucleus $\mathbf{T}_{n}$, then $\mathcal{T}^{\mathbf{M}}[\Phi()].(\mathbf{r})$ is an eigenfunction of the cascade $\mathbf{M} \mathbf{T}_{n} \mathbf{M}^{-1}$. This is immediately clear from the following: when the function $\mathcal{T}^{\mathbf{M}}[\Phi(\cdot)](\mathbf{r})$ is chosen as the input function of the cascade $\mathbf{M} \mathbf{T}_{n} \mathbf{M}^{-1}$, the inverse $\mathbf{M}$ system will convert it into $\Phi(\mathbf{r})$, which then acts as an eigenfunction of the nucleus $\mathbf{T}_{n}$, after which the forward $\mathbf{M}$ system transforms $\Phi(\mathbf{r})$ back again into the function $\mathcal{T}^{\mathbf{M}}[\Phi(\cdot)](\mathbf{r})$ with which we started.

Table 2. Seven Classes of Eigenvalue Distributions ${ }^{a}$

\begin{tabular}{l|lc|lc|c}
\hline$\lambda$ & $\mathcal{M}(s)$ or $\mathcal{H}(\ln s ; \cdot)$ & Section & $\mathcal{L}(\cdot)$ or $\mathcal{S}(\cdot)$ & Section & $\mathcal{F}(\theta ; \cdot)$ \\
\hline & $s, s^{-1}$ & $4 . \mathrm{A}$ & 1,1 & $4 . \mathrm{B}$ & $c^{i \theta}, e^{-i \theta b}$ \\
\hline $\mathcal{R}(\theta)$ & $s e^{i \theta}, s e^{-i \theta}, s^{-1} e^{-i \theta}, s^{-1} e^{i \theta}$ & $5 . \mathrm{A}$ & $e^{i \theta}, e^{i \theta}, e^{-i \theta}, e^{-i \theta}$ & $5 . \mathrm{B}$ & \\
$\mathcal{Z}$ & $s, s, s^{-1}, s^{-1}$ & $5 . \mathrm{C}$ & $1,1,1,1$ & $5 . \mathrm{C}^{c}$ & \\
& & & $\mathrm{Z}$ and $\{\mathcal{L}, S\}$ do not commute & & \\
\hline
\end{tabular}

${ }^{a}$ Seven classes of eigenvalue distributions $\lambda$ for first-order optical systems and their corresponding nuclei, composed of magnifiers $\mathcal{M}(s)$ or hyperbolic expanders $\mathcal{H}($ ln $s ; \cdot)$, lenses $\mathcal{L}(\cdot)$ or sections of free space $\mathcal{S}(\cdot)$, fractional Fourier transformers $\mathcal{F}(\theta ; \cdot)$, rotators $\mathcal{R}(\theta)$, and shearers $\mathcal{Z}$. Obvious concatenations of one-dimensional nuclei have not been stated explicitly.

${ }^{b}$ Nucleus for the one-dimensional class of Subsection 4.C is a fractional Fourier transformer $\mathcal{F}(\theta ; \cdot)$.

${ }^{c}$ Possible nuclei for the two-dimensional class of Subsection 5.D are a shearer $\mathcal{Z}$ followed by a lens $\mathcal{L}(\cdot)$ or followed by a section of free space $\mathcal{S}(\cdot)$. 


\section{A. Lens, Free Space, and Fractional Fourier}

\section{Transformer: Cases 2 and 3}

We recall that the lens $\mathcal{L}(f)$ and a section of free space $\mathcal{S}(z)$, i.e., the two one-dimensional nuclei presented in Subsection 4.B, can be described as

$$
\begin{gathered}
f_{o}(x)=f_{i}(x) \exp \left[-i \pi\left(1 / \lambda_{\mathrm{o}} f\right) x^{2}\right] \\
f_{o}\left(x_{o}\right)=\frac{1}{\sqrt{i \lambda_{\mathrm{o}} z}} \int_{-\infty}^{\infty} f_{i}\left(x_{i}\right) \exp \left[i \pi\left(\lambda_{\mathrm{o}} z\right)\left(x_{o}-x_{i}\right)^{2}\right] \mathrm{d} x_{i},
\end{gathered}
$$

respectively. The Dirac delta function $\delta(x-\xi)$ is an eigenfunction for any multiplication operator, with eigenvalue $\exp \left[-i \pi\left(1 / \lambda_{0} f\right) \xi^{2}\right]$ in the particular case of a lens $\mathcal{L}(f)$; and the harmonic signal (or plane wave) $\exp (i 2 \pi u x)$ is an eigenfunction for any convolution operator, with eigenvalue $\exp \left[i \pi\left(\lambda_{0} z\right) u^{2}\right]$ in the particular case of a section of free space $\mathcal{S}(z)$. With $\mathbf{M}$ being a $2 \times 2$ abcd matrix, we easily conclude that $\exp \left[i \pi b^{-1}\left(d x^{2}-2 \xi x\right)\right]$ is an eigenfunction of the system with ray transformation matrix $\mathbf{M} \mathbf{T}_{\mathcal{L}}(f) \mathbf{M}^{-1}$, which belongs to the class presented in Subsection 4.B. For example, for the particular case that $\mathbf{M}$ represents a Fourier transformer $\mathcal{F}(-\pi / 2 ; w)$, with $a=d=0, b=-w^{2}$, and $c=w^{-2}$ [Eq. (19)], we get the eigenfunctions $\exp \left(i 2 \pi \xi x / w^{2}\right)$ of a section of free space, which are plane waves, as expected.

We also recall ${ }^{16}$ that the Hermite-Gauss modes $\mathcal{H}_{k}(x ; w) \propto \exp \left(-\pi x^{2} / w^{2}\right) H_{k}(\sqrt{2 \pi} x / w) \quad\left[k=0,1, \ldots ; H_{k}(\cdot)\right.$ are the Hermite polynomials] are eigenfunctions of the fractional Fourier transformer $\mathcal{F}(\theta ; w)$, i.e., the unimodular-class nucleus presented in Subsection 4.C, with eigenvalues $\exp (-i k \pi / 2)$. Consequently, systems from the class of Subsection 4.C, with ray transformation matrix $\mathbf{M} \mathbf{T}_{\mathcal{F}}(\theta) \mathbf{M}^{-1}$, have as eigenfunctions the HermiteGauss-type modes $\mathcal{H}_{k}^{\mathbf{M}}(x ; w)=\mathcal{T}^{\mathbf{M}}\left[\mathcal{H}_{k}(\cdot ; w)\right](x)$ (Ref. 23), which can be found by letting the Hermite-Gauss modes $\mathcal{H}_{k}(x ; w)$ propagate through the abcd system.

\section{B. Magnifier, Rotator-Magnifier, and Rotator-Lens Combinations: Cases 1, 4, and 5}

While the input-output relationship for the magnifier $\mathcal{M}(s)$, i.e., the real-class nucleus [Subsection 4.A], reads

$$
|s|^{1 / 2} f_{o}(s x)=f_{i}(x),
$$

the complex-quartet-class nucleus $\mathcal{M}(s, s) \mathcal{R}(\theta)$ [Subsection 5.A] and the double-unimodular-class nucleus [Subsection 5.B] can easily be described in polar coordinates [Eqs. (37) and (41)]. While powers $x^{k}$ are evidently eigenfunctions of the real-class nucleus $\mathcal{M}(s)$ with eigenvalues $|s|^{-1 / 2} s^{-k}$, signals of the form $r^{k} \exp (\operatorname{im} \varphi)$ are eigenfunctions of the complex-quartet-class nucleus $\mathcal{M}(s, s) \mathcal{R}(\theta)$ with eigenvalues $s^{-1 / 2} s^{-k} \exp (\operatorname{im} \theta)$, and signals of the form $\delta(r-\rho) \exp (i m \varphi)$ are eigenfunctions of the doubleunimodular-class nucleus $\mathcal{L}(f, f) \mathcal{R}(\theta)$ with eigenvalues $\exp (i m \theta) \exp \left[-i \pi\left(1 / \lambda_{0} f\right) \rho^{2}\right]$.

From the powers $x^{k}$ we derive that the eigenfunctions of the system with ray transformation matrix $\mathbf{M} \mathbf{T}_{\mathcal{M}}(s) \mathbf{M}^{-1}$, which belongs to the class presented in Subsection 4.A, take the same mathematical form as the Hermite-Gauss-type modes but with complex arguments. For example, for the particular case that $\mathbf{M}$ represents a fractional Fourier transformer $\mathcal{F}(-\pi / 4 ; w)$, with $a=d$
$=1 / \sqrt{2}, b=-w^{2} / \sqrt{2}$, and $c=w^{-2} / \sqrt{2}$ [Eq. (16)], we get the eigenfunctions $\mathcal{H}_{k}\left(x ; \sqrt{i w^{2}}\right) \propto \exp \left(i \pi x^{2} / w^{2}\right) H_{k}\left(\sqrt{2 \pi} x / \sqrt{i w^{2}}\right)$ of the hyperbolic expander. ${ }^{24}$ Note that these eigenfunctions show a chirplike behavior in contrast to the Gaussian-like behavior of the normal Hermite-Gauss modes (see also Ref. 10, Sections V.B and V.C).

Eigenfunctions of the systems that belong to the classes presented in Subsections 5.A and 5.B can be found along the same lines, using the functions $r^{k} \exp (\operatorname{im} \varphi)$ and $\delta(r-\rho) \exp (i m \varphi)$, respectively. As a first example, let us determine eigenfunctions of the system with ray transformation matrix $\mathbf{M} \mathbf{T}_{\mathcal{L} \mathcal{R}}(\theta ; f) \mathbf{M}^{-1}$, which belongs to the class presented in Subsection 5.B, for the special case that $\mathbf{M}$ is isotropic (i.e., with submatrices $a \mathbf{I}, b \mathbf{I}, c \mathbf{I}$, and $d \mathbf{I}$ ). We therefore have to calculate the integral

$$
\begin{aligned}
\int_{0}^{\infty} \int_{0}^{2 \pi} \delta\left(r_{i}-\rho\right) \exp \left(i m \varphi_{i}\right) \\
\quad \times \exp \left[i \pi b^{-1}\left(a r_{i}^{2}-2 r_{i} r \cos \left(\varphi_{i}-\varphi\right)+d r^{2}\right)\right] r_{i} \mathrm{~d} r_{i} \mathrm{~d} \varphi_{i} \\
=\rho \exp \left[i \pi b^{-1}\left(a \rho^{2}+d r^{2}\right)\right] \exp (i m \varphi) \\
\quad \times \int_{0}^{2 \pi} \exp \left[-i 2 \pi b^{-1} \rho r \cos \left(\varphi_{i}-\varphi\right)+i m\left(\varphi_{i}-\varphi\right)\right] \mathrm{d} \varphi_{i} \\
=\rho \exp \left[i \pi b^{-1}\left(a \rho^{2}+d r^{2}\right)\right] \\
\quad \times \exp (i m \varphi) 2 \pi(-i)^{m} J_{m}\left(2 \pi b^{-1} \rho r\right),
\end{aligned}
$$

and we conclude that functions of the form $\exp \left(i \pi b^{-1} d r^{2}\right) \exp (i m \varphi) J_{m}\left(2 \pi b^{-1} \rho r\right)$ are eigenfunctions of this system. As a second example, we determine eigenfunctions of the systems with ray transformation matrix $\mathbf{M} \mathbf{T}_{\mathcal{M R}}(\theta, s) \mathbf{M}^{-1}$, which belongs to the class presented in Subsection 5.A, for the special case again that $\mathbf{M}$ is isotropic. We now have to calculate the integral

$$
\begin{aligned}
\int_{0}^{\infty} & \int_{0}^{2 \pi} r_{i}^{k} \exp \left(i m \varphi_{i}\right) \\
& \times \exp \left[i \pi b^{-1}\left(a r_{i}^{2}-2 r_{i} r \cos \left(\varphi_{i}-\varphi\right)+d r^{2}\right)\right] r_{i} \mathrm{~d} r_{i} \mathrm{~d} \varphi_{i} \\
= & \exp \left(i \pi b^{-1} d r^{2}\right) \exp (i m \varphi) 2 \pi(-i)^{m} \\
& \times \int_{0}^{\infty} r_{i}^{k+1} \exp \left(i \pi b^{-1} a r_{i}^{2}\right) J_{m}\left(2 \pi b^{-1} r_{i} r\right) \mathrm{d} r_{i} .
\end{aligned}
$$

\section{Shearer-Magnifier and Shearer-Lens Combinations:} Cases 6 and 7

The same can be done for the systems that belong to the classes presented in Subsections 5.C and 5.D. However, proper eigenfunctions of the nuclei for these classes are still to be found.

\section{CONCLUSION}

We have presented a classification of one- and twodimensional real symplectic matrices, based on the distribution of the eigenvalues of the matrix and on whether or not it can be diagonalized. Subsequently, for each class we have formulated simple lossless first-order optical systems that can be considered as a nucleus for this class: 
the nucleus is described by as few parameters as possible and its ray transformation matrix satisfies the properties of realness and symplecticity. We have thus suggested: (1) the magnifier (and a hyperbolic expander), (2) the lens (and a section of free space), and (3) a fractional Fourier transformer as nuclei for the three classes that arise in the one-dimensional case. For the two-dimensional case we have suggested, in addition to the obvious concatenations of one-dimensional nuclei, the four inherently twodimensional combinations: (4) an isotropic magnifier (or hyperbolic expander) with a rotator, (5) an isotropic lens (or section of free space) with a rotator, (6) an isotropic magnifier (or hyperbolic expander) with a shearing operator, and (7) an isotropic lens (or section of free space) with a shearing operator.

Any first-order optical system belongs to one of the classes described in this paper and is similar (in the sense of matrix similarity of the ray transformation matrices) to the corresponding nucleus. We have shown how knowledge of the nucleus may be helpful in finding eigenfunctions of the corresponding class of first-order optical systems and of the linear canonical integral transformation that is associated to each system: one only has to find eigenfunctions of the (simple) nucleus and to determine how these functions propagate through a first-order optical system.

The nuclei of those systems whose eigenvalues are unimodular (cases 2-2, 2-3, 3-3, 5, and 7) might be useful for the design of stable optical resonators.

\section{ACKNOWLEDGMENTS}

M. J. Bastiaans appreciates the hospitality at Universidad Complutense de Madrid. T. Alieva acknowledges the Spanish Ministry of Education and Science (project TEC 2005-02180/MIC).

M. J. Bastiaans' e-mail address is m.j.bastiaans @tue.nl. T. Alieva's e-mail address is talieva@fis.ucm.es.

\section{REFERENCES}

1. R. K. Luneburg, Mathematical Theory of Optics (U. of California Press, 1966)

2. A. Vander Lugt, "Operational notation for the analysis and synthesis of optical data-processing systems," Proc. IEEE 54, 1055-1063 (1966).

3. H. J. Butterweck, "General theory of linear, coherent optical data-processing systems," J. Opt. Soc. Am. 67, 60-70 (1977).
4. M. Nazarathy and J. Shamir, "First-order optics-a canonical operator representation: lossless systems," J. Opt. Soc. Am. 72, 356-364 (1982).

5. J. W. Goodman, Introduction to Fourier Optics, 2nd ed. (McGraw-Hill, 1996).

6. K. B. Wolf, Geometric Optics on Phase Space (Springer, 2004).

7. S. A. Collins, Jr., "Lens-system diffraction integral written in terms of matrix optics," J. Opt. Soc. Am. 60, 1168-1177 (1970).

8. M. Moshinsky and C. Quesne, "Linear canonical transformations and their unitary representations," J. Math. Phys. 12, 1772-1780 (1971).

9. K. B. Wolf, Integral Transforms in Science and Engineering (Plenum, 1979).

10. S. C. Pei and J. J. Ding, "Eigenfunctions of linear canonical transform," IEEE Trans. Signal Process. 50, 11-26 (2002).

11. V. Namias, "The fractional order Fourier transform and its applications to quantum mechanics," J. Inst. Math. Appl. 25, 241-265 (1980).

12. A. C. McBride and F. H. Kerr, "On Namias' fractional Fourier transforms," IMA J. Appl. Math. 39, 159-175 (1987).

13. A. W. Lohmann, "Image rotation, Wigner rotation, and the fractional Fourier transform,” J. Opt. Soc. Am. A 10, 2181-2186 (1993)

14. D. Mendlovic and H. M. Ozaktas, "Fractional Fourier transforms and their optical implementation: I," J. Opt. Soc. Am. A 10, 1875-1881 (1993).

15. H. M. Ozaktas and D. Mendlovic, "Fractional Fourier transforms and their optical implementation: II," J. Opt. Soc. Am. A 10, 2522-2531 (1993).

16. H. M. Ozaktas, Z. Zalevsky, and M. A. Kutay, The Fractional Fourier Transform with Applications in Optics and Signal Processing (Wiley, 2001).

17. F. R. Gantmacher, The Theory of Matrices (Chelsea, 1974-1977).

18. M. J. Bastiaans and T. Alieva, "First-order optical systems with unimodular eigenvalues," J. Opt. Soc. Am. A 23, 1875-1883 (2006).

19. W. W. Lin, V. Mehrmann, and H. Xu, "Canonical forms for Hamiltonian and symplectic matrices and pencils," Linear Algebr. Appl. 302-303, 469-533 (1999).

20. R. Simon and K. B. Wolf, "Structure of the set of paraxial optical systems," J. Opt. Soc. Am. A 17, 342-355 (2000).

21. R. Simon and N. Mukunda, "Iwasawa decomposition in first-order optics: universal treatment of shape-invariant propagation for coherent and partially coherent beams," J. Opt. Soc. Am. A 15, 2146-2155 (1998).

22. T. Alieva and M. J. Bastiaans, "Alternative representation of the linear canonical integral transform," Opt. Lett. 30, 3302-3304 (2005).

23. M. J. Bastiaans and T. Alieva, "Generating function for Hermite-Gaussian modes propagating through first-order optical systems," J. Phys. A 38, L73-L78 (2005).

24. M. J. Bastiaans and T. Alieva, "First-order optical systems with real eigenvalues," Opt. Commun. 272, 52-55 (2007). 